\title{
Prevalencia de sobrepeso y obesidad en escolares preadolescentes del municipio de Aguascalientes
}

De Alba-López ZC, Del Real-Cajero J, Esparza-Flores MI, García-González RA, Herrera-Ruvalcaba JE, Juárez-Ávila M, Malo-Martínez NP, Márquez-Reyes JN, Terrones-Saldívar MC, Rosas-Cabral A.

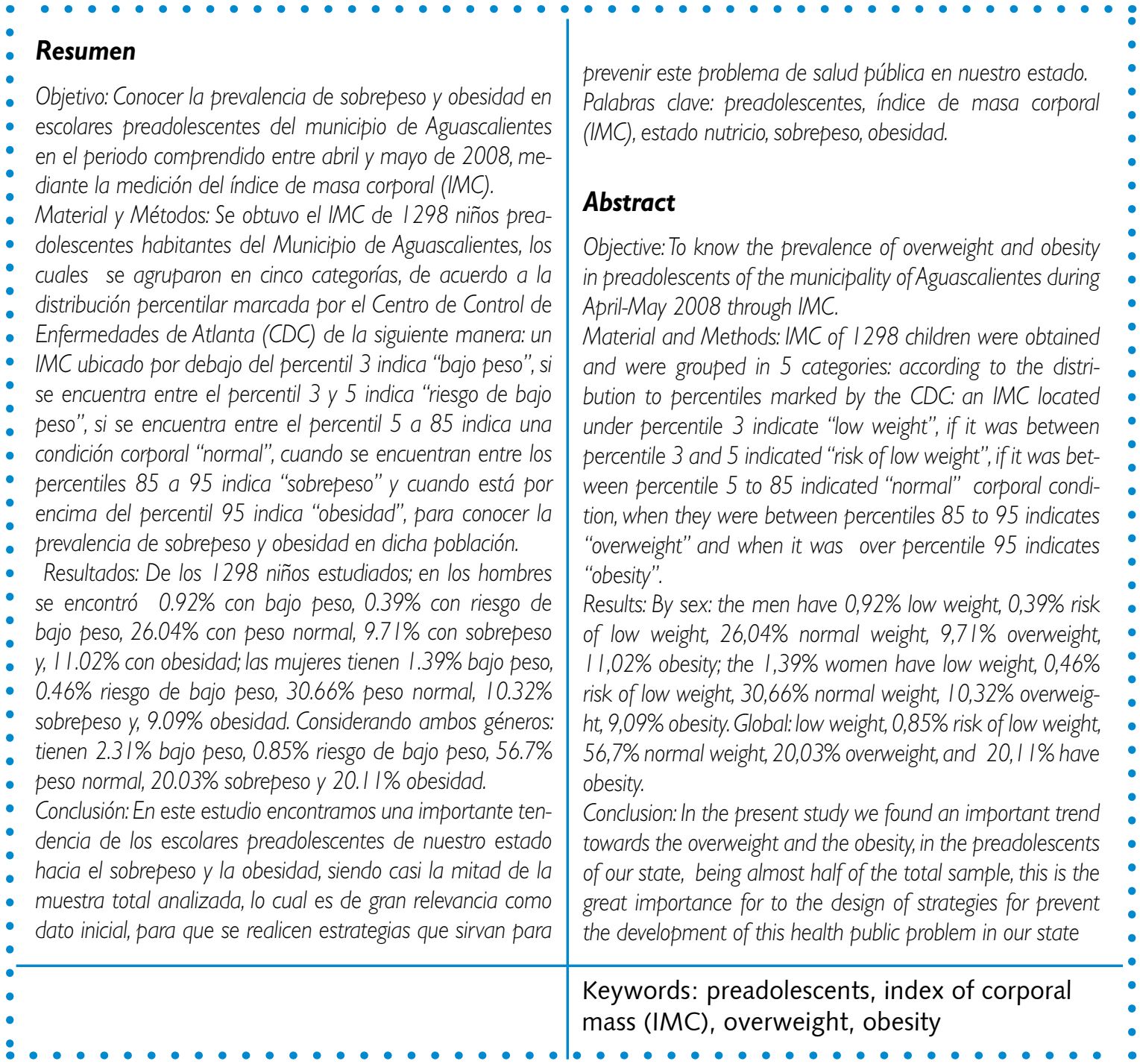




\section{Introducción}

Una correcta nutrición es deseable a cualquier edad, pero la población escolar despierta un especial interés en el estudio nutricional si consideramos aspectos biológicos, psicológicos y sociales. La alimentación constituye un elemento fundamental para un buen desarrollo, sobre todo en la primera infancia y en la etapa escolar. Es precisamente en los primeros años de vida, donde hay que empezar a educar sobre una alimentación sana, evitando errores o modas que puedan llevar al individuo en la edad adulta a tener problemas irreversibles ${ }^{1}$.

En los últimos años, en México se han desarrollado múltiples programas para recabar datos antropométricos que permitan evaluar el estado nutricio de la población pediátrica, debido a que enfermedades crónico degenerativas, tales como la diabetes mellitus y la hipertensión arterial sistémica, aparecen cada vez a edades más tempranas y dado que constituyen las primeras causas de morbi mortalidad en nuestro país, es indispensable entablar medidas encaminadas a la detección temprana del sobrepeso y la obesidad como precursores importantes de estos padecimientos ${ }^{2}$; a largo plazo, un adolescente obeso tiene 1.8 veces mayor mortalidad por todas las causas y 2.3 por enfermedad coronaria ${ }^{3}$. El deterioro del estado nutricional afecta a todo el organismo y eleva la vulnerabilidad del niño a las infecciones, lo que incrementa la mortalidad infantil. Es por ello que la evaluación del estado nutricional en el niño es un componente esencial de atención médica y un elemento básico para determinar el estado de salud de cada niño. Asimismo, el bajo peso conduce a un mayor riesgo de adquirir diversos estados patológicos, siendo los principales las enfermedades infectocontagiosas ${ }^{4}$.

La mala nutrición (tanto la desnutrición como el exceso en la ingesta) tiene causas complejas que involucran determinantes biológicos, socioeconómicos y culturales. La desnutrición en el niño es el resultado directo de una dieta inadecuada, en cantidad o calidad, y del efecto acumulativo de episodios repetidos de enfermedades infecciosas o de otros padecimientos. Estos factores tienen su origen en el acceso insuficiente a alimentos nutritivos, servicios de salud deficientes, saneamiento ambiental inadecuado y prácticas inapropiadas de cuidado en el hogar ${ }^{5}$.

La prevalencia del sobrepeso y la obesidad está aumentada en las poblaciones infantiles en todo el mundo. La obesidad en los niños tiene consecuencias significativas en la salud a largo plazo. En el 2005 había en todo el mundo al menos 20 millones de menores de 5 años con sobrepeso. Aunque antes se consideraba un problema exclusivo de los países de altos ingresos, el sobrepeso y la obesidad están aumentando espectacularmente en los países de ingresos bajos y medios, sobre todo en el medio urbano ${ }^{6}$.

La obesidad es el resultado de un desequilibrio entre la ingestión y el gasto energético. Este desequilibrio es consecuencia de la 
ingestión de dietas con alta densidad energética y bajas en fibra, bebidas azucaradas, en combinación con una escasa actividad física. Esta última se ha asociado a la urbanización, al crecimiento económico y a los cambios en la tecnología para la producción de bienes y servicios, así como a los estilos de vida y de recreación ${ }^{7}$.

Actualmente el IMC es el indicador de primera elección para el diagnóstico de obesidad en niños. Su cálculo es sencillo, reproducible y económico, además, existe correlación adecuada con la cantidad de grasa corporal ${ }^{8}$. Las limitaciones se relacionan con las variaciones derivadas de la edad, el sexo y la maduración, tanto entre distintas poblaciones como en una misma. El patrón de referencia CDC 2000 es una actualización del formulado por la Organización Mundial de la Salud, que incluye tablas y gráficas de IMC para la edad y sexo de los dos a los 20 años de edad ${ }^{9}$.

De acuerdo con la Encuesta Nacional de Salud y Nutrición 2006 (ENSANUT 2006), la prevalencia nacional combinada de sobrepeso y obesidad en niños de 5 a 11 años, fue de alrededor de $26 \%$ para ambos sexos, $26.8 \%$ en niñas y $25.9 \%$ en niños. De la misma forma, uno de cada tres hombres o mujeres adolescentes (12 a 19 años) tiene sobrepeso $u$ obesidad. No hay claras tendencias del sobrepeso y la obesidad en relación con la edad, salvo una ligera tendencia de mayor obesidad a mayor edad en el caso de las mujeres, en quienes la prevalencia de sobrepeso fue de 23.3 $\%$ y de obesidad de $33.3 \%{ }^{10}$. En el municipio de Aguascalientes no se ha realizado algún estudio para conocer el estado nutricio de los preadolescentes, lo cual es de vital importancia para modificar el panorama epidemiológico actual y por lo tanto ayudar a disminuir la incidencia de los principales problemas de salud de la población pediátrica y de manera prospectiva mejorar también, el estado de salud de la población adulta de nuestro estado, por tal motivo realizamos el presente trabajo para conocer la prevalencia de sobrepeso y obesidad en los preadolescentes del Municipio de Aguascalientes.

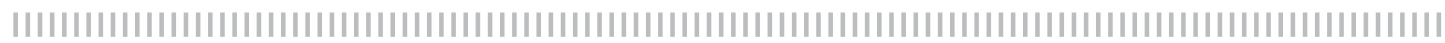

\section{Material y métodos}

Se realizó un estudio observacional, descriptivo y transversal, mediante muestreo multietápico y aleatorio, se determinó una muestra total de 1340 niños, de los cuales se eliminaron a 41 niños por ser mayores de 12 años (entre 13-15 años) y una niña de 9 años, ya que la OMS y la Secretaria de Salud de México define como preadolescencia o adolescencia temprana al periodo comprendido entre los 10 a los 12 años, quedando con un total de 1298 niños, inscritos en quinto y sexto grado de las escuelas de educación primaria de todo el Municipio de Aguascalientes.

Se obtuvo el peso y la talla por medio de un estadímetro y báscula integrados de fabricación nacional (Dyna-Top modelo E1) con capacidad de medir talla hasta de 2 metros, con precisión de $1 \mathrm{~mm}$., el peso fue medido hasta los 100 gr. más próximos. Se calculó el Índice de Masa Corporal 
$\left(I M C=\right.$ (peso en $\mathrm{kg} /$ talla en $\left.\mathrm{m}^{2}\right)$ para cada individuo y con base a este parámetro, se utilizó el programa de estadística SPSS versión 14, donde se agruparon en cinco categorías según la distribución percentilar marcada por el CDC de la siguiente manera: un IMC ubicado por debajo del percentil 3 indica "bajo peso", si se encuentra entre el percentil 3 y 5 indica "riesgo de bajo peso", si se encuentra entre el percentil 5 a 85 indica una condición corporal "normal", cuando se encuentran entre los percentiles 85 a 95 indica "sobrepeso" y cuando está por encima del percentil 95 indica "obesidad".

\section{| | | | | | | | | | | | | | | | | | | | | | | | | | | | | | | | | | | | | | | | | | | | | | | | | | | | | | | | | | | | | | | | | | | | | | | | | | | | | | | | | | | | | | | | | | | | | | | | | | | | ||}

\section{Resultados}

Se estudiaron 1298 niños, de los cuales fueron 624 niños $(48.07 \%)$ y 674 niñas $(51.92 \%)$ con edad promedio de 11 años. En los niños $0.92 \%$ presentan bajo peso, $0.39 \%$ riesgo de bajo peso, $26.04 \%$ presentan un peso normal dentro de los rangos normales según la distribución del CDC, $9.71 \%$ con sobrepeso, $11.02 \%$ tienen obesidad, hay una tendencia al exceso de peso del $20.73 \%$ más que al bajo peso de $1.31 \%$. (Tabla 1)

En las niñas $1.39 \%$ presentan bajo peso, $0.46 \%$ riesgo de bajo peso, $30.66 \%$ presentan un peso normal dentro de los rangos normales según la distribución del
CDC, $10.32 \%$ con sobrepeso, $9.09 \%$ tienen obesidad (Ver Gráfica 1). En los resultados globales sin tomar en cuenta el sexo, tenemos que el $2.31 \%$ tienen bajo peso, $0.85 \%$ riesgo de bajo peso, el $56.7 \%$ peso normal, $20.03 \%$ sobrepeso y $20.11 \%$ obesidad.

Al comparar el porcentaje de niños que se distribuyeron entre las categorías de peso bajo (bajo peso mas riesgo de bajo peso) versus exceso de peso (considerando para tal situación a los que caen en sobrepeso y obesidad) se observó que el $40.14 \%$, caen en la categoría de exceso de peso contra el $3.16 \%$ de niños en peso subnormal, (Ver gráfica 2).

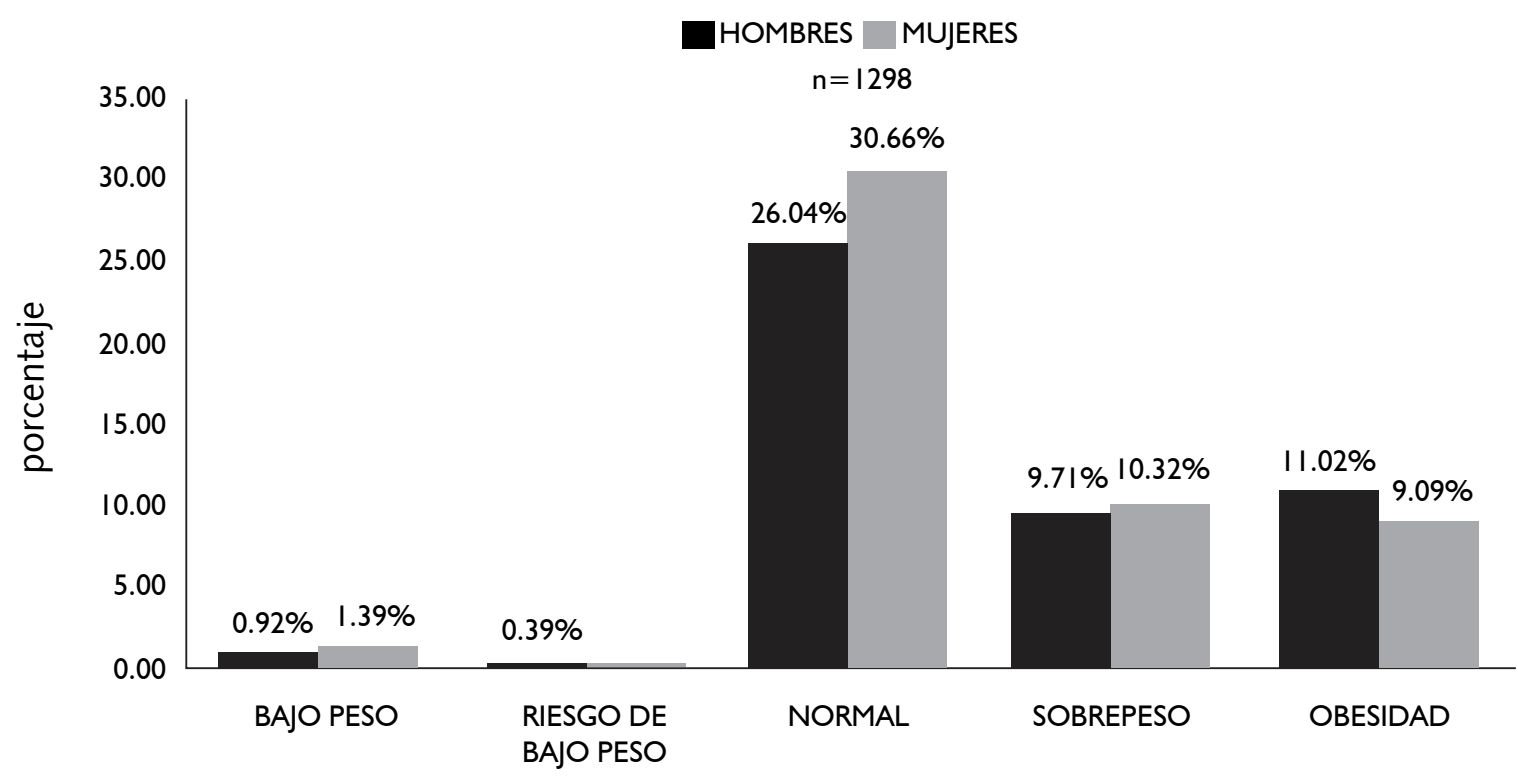

Gráfica 1. Distribución según género y categoría del IMC. 


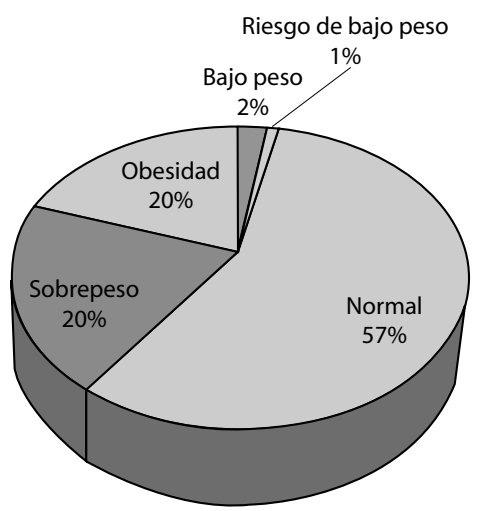

Gráfica 2. Distribución global por categorías deacuerdo al IMC

\section{Discusión}

El deterioro del estado nutricio afecta a todo el organismo y eleva la vulnerabilidad del niño a las infecciones, lo que incrementa la mortalidad infantil. Es por ello que su evaluación es un componente esencial de atención médica y un elemento básico para determinar el estado de salud de cada niño ${ }^{11-12}$.

En los resultados obtenidos se puede observar que en los preadolescentes masculinos el porcentaje de bajo peso es menor que el de las mujeres, así mismo, llama la atención que tienen mayor proporción de obesidad que las mujeres, aunque no son estadísticamente diferentes. En el caso de riesgo de bajo peso es realmente poco significativa la diferencia entre ambos sexos. También es importante notar que las mujeres tienen bajo peso y riesgo de bajo peso mayor que los hombres lo que es importante para tomar medidas y evitar efecto "matrushka"14.

A pesar de que el diagnóstico de desnutrición requiere de la utilización de otros parámetros ${ }^{13}$, es necesario detectar y estudiar detalladamente a los niños con bajo peso, para poder diferenciar entre un retraso carencial del crecimiento o simplemente la tendencia esperada de acuerdo a la constitución esperada en función de su carga genética.

Por otro lado, el sobrepeso y la obesidad, así como las enfermedades derivadas o propiciadas a partir de estas alteraciones metabólicas, son actualmente el problema de salud pública más apremiante que enfrenta nuestro país. En su aparición intervienen la carga genética de cada individuo, el estilo de vida sedentario, el mayor consumo calórico en la dieta y la menor capacidad de saciar esta demanda, son los principales factores que explican la obesidad. El sedentarismo y un nivel bajo de actividad física se acompaña de un bajo nivel de oxidación de la grasa y éste es un factor importante de riesgo para una mayor ganancia de peso ${ }^{15}$.

El sobrepeso y la potencial obesidad en el niño pueden impactar su salud física y psicológica a corto, mediano y largo plazo. La hiperlipidemia, hipertensión y tolerancia anormal a la glucosa ocurren con mayor frecuencia en niños y adolescentes obesos. Otros trastornos comunes son los problemas ortopédicos, la disminución de la autoestima y el deterioro de la percepción de la imagen corporal ${ }^{6}$.

Como se refiere en el reciente reporte de Bonvecchio y colaboradores, la epidemia de sobrepeso y obesidad está afectando a los niños mexicanos de todos los grupos de edad, regiones y condiciones sociales. Aquí es relevante notar que este estudio representa una clara evidencia de que dicho problema también está presente en nuestro estado y que afecta a un grupo 
de edad en el cual aún es factible tomar medidas para modificar los estilos de vida y dietéticos que conduzcan a una distribu- ción de peso más saludable y que las mismas medidas sean culturalmente aceptables y efectivas ${ }^{15}$.

\section{Conclusiones}

En el presente estudio encontramos que casi la mitad de nuestra muestra de preadolescentes escolares, habitantes del municipio de Aguascalientes, tiene obesidad y sobrepeso, similar a la reportada en el resto del país ${ }^{16}$. Lo cual hace necesario establecer estrategias que involucren el ámbito familiar y escolar para apoyar al escolar en el cambio de hábitos nutricionales y de actividad física.

Agradecimientos

A la subdirectora del IEA, a los coordinadores y maestros de las diferentes escuelas primarias participantes en el estudio, por mostrar interés y facilitarnos lo necesario para nuestro trabajo, a nuestros maestros como asesores y excelentes guías, al comité de LUX MÉDICA, por sus críticas constructivas para el mejoramiento de este trabajo.

\section{Bibliografía}

1. Castañeda R. E. et al. Estado nutricional en el estado de Hidalgo. Revista de Endocrinología y Nutrición. 2002; 10(4):201-205.

2. Kenneth Lee Jones. Role of Obesity in Complicating and Confusing the Diagnosis and Treatment of Diabetes in Children; Pediatrics. 2008; 121; 361-368.

3. UNICEF. The State of the World's Children 1998. Oxford: Oxford University Press, 1998.

4. Weiss R., Dziura J., Burgert T. S., Tamborlane W. V., Taksali S. E., Yeckel C. W. Allen K., López M., Savoye M., Morrison J., Sherwin R. S., Caprio S., Obesity and the Metabolic Syndrome in Children and Adolescents, NEJM. 2004; 350: 2362-74.

5. Popkin B. An overview on the nutrition transition and its health implications: The Bellagio meeting. Public Health Nutr. 2002; 5(1A): 93-103.

6. Romero V. E., Campollo R. O., Castro H. J. F., Cruz O. R., Vásquez G. E., Hábitos de alimentación e ingestión de calorías en un grupo de niños y adolescentes obesos: Boletín Médico Hospital Infantil de México. 2006;63.

7. Must $A$, Jacques PF, Dallal GE, Bajema CJ, Dietz WH. Long-term morbidity and mortality of overweight adolescents. A follow-up of the Harvard Growth Study of 1922 to 1935. NEJM 1992;327(19):1379-1380.

8. Noevius $M$, Linné $Y$, Barkeling $B$, Rösner $S$. Discre- pancies between classification systems of childhood obesity. Obes Rev 2004; 5:105-114.

9. Centers for Disease Control and Prevention. 2000 CDC Growth Charts: United States. Atlanta, GA: Centers for Disease Control and Prevention; 2000. Disponible en http://www.cdc.gov/growthcharts

10. Encuesta Nacional de Nutrición y Salud 2006. Instituto Nacional de Salud Pública, Secretaria de Salud, México.

11. Lifshitz F, Moisés N. Growth failure. A complication of dietary treatment of hipercholesterolemia. Am J Dis Child. 1989; 143: 537-42.

12. Pugliese M., Lifshitz F., Grad G., Fort P. Fear of obesity: a cause of short stature and delay puberty. NEJM,.1983; 309: 513-518.

13. Saucedo Molina TJ, Gómez-Peresmitre G. Validación del índice nutricional en preadolescentes mexicanos con el método de sensibilidad y especificidad. Salud Publica Mex 1998; 40:392-397.

14. Flores Huerta S. Desnutrición energético-proteínica. Games E. Troconis T. $7^{\circ}$ Edición. México, D.F. Méndez Editores. 2006. Págs. 143-155.

15. Bonvecchio A, Safdie $M$, Monterrubio EA, Gust T, Villalpando S, Rivera JA. Salud Pública de Mex. 2009;51:Supl. 4:S586-S594

16. Ortiz H. L., Estado nutricio en adolescentes de una población suburbana de la ciudad de México, Revista Mexicana Pediátrica 2003; 70(3); 109-117.. 